Article

\title{
Doppler-Based Acoustic Gyrator
}

\author{
Farzad Zangeneh-Nejad and Romain Fleury * \\ Laboratory of Wave Engineering, École Polytechnique Fédérale de Lausanne (EPFL), 1015 Lausanne, \\ Switzerland; farzad.zangenehnejad@epfl.ch \\ * Correspondence: romain.fleury@epfl.ch; Tel.: +41-412-1693-5688
}

Received: 30 May 2018; Accepted: 2 July 2018; Published: 3 July 2018

check for updates

\begin{abstract}
Non-reciprocal phase shifters have been attracting a great deal of attention due to their important applications in filtering, isolation, modulation, and mode locking. Here, we demonstrate a non-reciprocal acoustic phase shifter using a simple acoustic waveguide. We show, both analytically and numerically, that when the fluid within the waveguide is biased by a time-independent velocity, the sound waves travelling in forward and backward directions experience different amounts of phase shifts. We further show that the differential phase shift between the forward and backward waves can be conveniently adjusted by changing the imparted bias velocity. Setting the corresponding differential phase shift to 180 degrees, we then realize an acoustic gyrator, which is of paramount importance not only for the network realization of two port components, but also as the building block for the construction of different non-reciprocal devices like isolators and circulators.
\end{abstract}

Keywords: non-reciprocal acoustics; gyrators; phase shifters; Doppler effect

\section{Introduction}

Microwave phase shifters are two-port components that provide an arbitrary and variable transmission phase angle with low insertion loss [1-3]. Since their discovery in the 19th century, such phase shifters have found important applications in devices such as phased array antennas and receivers [4,5], beam forming and steering networks [6,7], measurement and testing systems [8,9], filters [10,11], modulators [12,13], frequency up-convertors [14], power flow controllers [15], interferometers [16], and mode lockers [17].

Various types of microwave phase shifters have been proposed and demonstrated over years, all of which can be fundamentally categorized into two groups: reciprocal and non-reciprocal phase shifters. Non-reciprocal phase shifters providing different phase shift for forward and backward waves, however, are of more importance especially when it comes to the realization of devices like gyrators [18], isolators [19], and circulators [20], being critical for radar systems and networks.

In its most traditional form, a non-reciprocal microwave phase shifter is composed of a rectangular waveguide partially filled with a ferrite slab submitted to a magneto-static bias field [1] (see Figure 1a). When the bias field is zero, the ferrite is demagnetized and the structure provides a reciprocal phase shift of $\varphi_{0}$ for instance, being proportional to the length of the waveguide, for both forward and backward propagation. However, when the bias field is increased from zero, the ferrite slab starts magnetizing, giving rise to distinct and continuously variable phase shifts of $\varphi_{1}$ and $\varphi_{2}$ for forward and backward waves, respectively.

In contrast to electromagnetism, realizing a non-reciprocal phase shifter in acoustics is challenging as sound waves only weakly interact with magnetic fields [21-29]. Recently, proposals to break the conventional wisdom of sound reciprocity have been reported, of which most rely on non-linear mechanisms [30-33]. Despite their applicability, these nonlinear structures accompany with unwanted 
features such as high-power consumption and geometrical bulky configuration, reducing their practical worth.
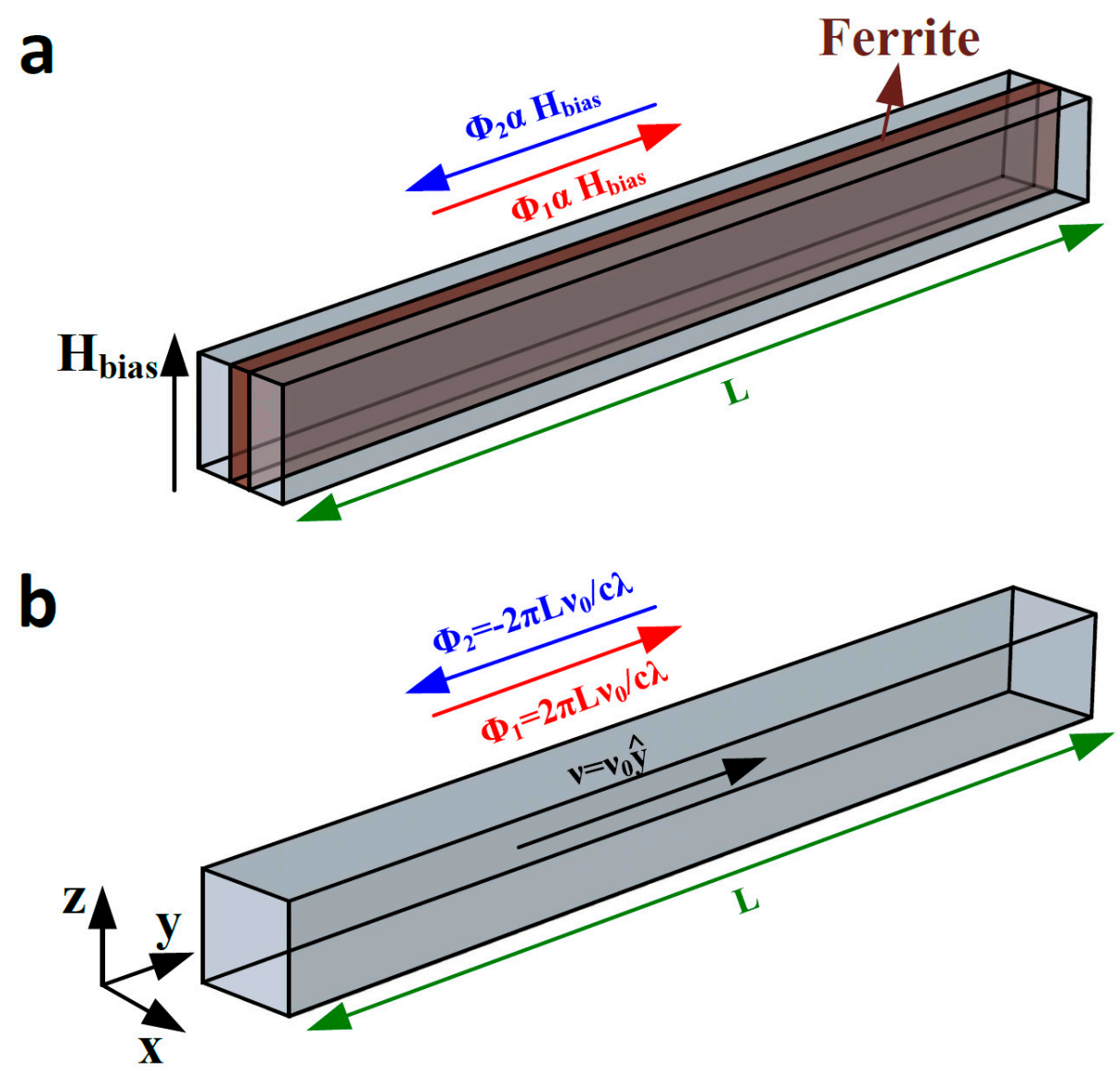

Figure 1. Concept of non-reciprocal phase shifters. (a) Schematic of a typical non-reciprocal phase shifter in microwave engineering. The device is composed of a waveguide that is partially filled with a ferrite slab on which a constant magnetic bias field is applied. The amount of differential phase shift between the forward and backward waves can be controlled by changing the magnetic field. (b) Acoustic equivalent of the microwave phase shifter shown in panel a. The structure consists of an acoustic waveguide that is filled with a fluid on which a velocity motion of $v_{0}$ is applied. The waveguide provides different phase shifts of $\varphi_{1}$ and $\varphi_{2}$ for forward and backward transmission, respectively.

A recent breakthrough, however, has opened exciting venues to break the reciprocity of acoustic waves in fully linear manners. In [22], it was shown that applying a time-independent bias velocity to stationary fluids allows one to strongly break the time reversal symmetry and to induce acoustic non-reciprocity. This salient feature was then leveraged to demonstrate a subwavelength circulator in airborne acoustics, allowing audible sound to be coupled to a certain port, while isolating transmission in another.

On the basis of this idea, here we first demonstrate a fully linear non-reciprocal acoustic phase shifter. This is achieved in a simple acoustic waveguide that is filled with air on which a constant bias velocity is applied. Through analytical analysis and numerical simulations, we show how the proposed structure provides different phase shifts for forward and backward transmission. We further show that the associated differential phase shift can be simply tuned by changing the bias velocity. By setting a fluid bias velocity that corresponds to a differential phase shift of 180 degrees, we then implement an acoustic gyrator, which is an important two port component serving as the building block for a large variety of non-reciprocal acoustic devices such as isolators and circulators. We believe 
that our finding opens up exciting frontiers in the field of modern acoustic engineering [34-67] for the design and realization of various innovative components, such as phase modulators, beam forming devices, switches, and frequency convertors.

\section{Acoustic Phase Shifter}

In this section, we focus our attention on realizing a non-reciprocal acoustic phase shifter that brings about different phase shifts for forward and backward waves. As a starting point, consider the geometry represented in Figure $1 \mathrm{~b}$ consisting of an acoustic waveguide filled with air on which a velocity of $v=v_{0} \hat{y}$ is assumed to be imparted. The overall length of the waveguide is supposed to be $L=7 \lambda=73 \mathrm{~cm}$, where $\lambda$ is the wavelength of the operation. When the fluid inside the waveguide is stationary, i.e., when $v_{0}=0$, the signals travelling from the left to the right side of the waveguide acquire an overall phase shift of zero, as the waveguide length is a multiple of the wavelength. Similarly, sound waves injected into the right side of the waveguide are subject to an effective phase shift of zero when reaching the left side, which is consistent with the reciprocity of the device. When assuming a non-zero bias velocity motion $\left(v_{0} \neq 0\right)$, however, the frequency of the signals propagating from the left to the right is Doppler shifted by an amount of $\Delta f=-v_{0} / \lambda$. Consequently, they obtain an additional phase shift of $\varphi_{1}=\frac{2 \pi v_{0} L}{c \lambda}$ ( $c$ is the speed of sound in the air) during their propagation (the red arrow in the figure). Figure $2 \mathrm{a}$ (the red line) illustrates the variation of $\varphi_{1}$ versus the fluid velocity $v_{0}$. The figure reveals that by changing the bias velocity, one can readily tune the overall phase shift that a signal obtains when passing through the waveguide. However, this is not something special by itself, as the same effect could be achieved by changing the length of the waveguide. The salient feature of the proposed structure becomes clear when we look at the propagation of the waves that travel in the opposite direction. In contrast to the previous case, the frequency of these types of signals is upshifted by an amount of $\Delta f=v_{0} / \lambda$ with respect to the static frequency $f_{0}=c / \lambda$. The corresponding Doppler phase shift is therefore $\varphi_{2}=-\frac{2 \pi v_{0} L}{c \lambda}$ in this case, which is negative of $\varphi_{1}$. Figure $2 \mathrm{a}$ (the blue line) plots the dependence of $\varphi_{2}$ on the fluid motion velocity $v_{0}$. It is evident that the motion of the fluid within the waveguide results in different phase shifts for forward and backward sound waves, making the structure non-reciprocal in phase. Notably, it was not possible to achieve such non-reciprocal behavior by solely changing the length of the waveguide. We further note that since there is no geometrical detour causing reflection or refraction, and since the amount of sound attenuation is quite low at the frequency of operation, the amplitude of the transmitted signals maintains almost the same value, regardless of the propagation direction. One can therefore express the scattering matrix of the waveguide as:

$$
\mathrm{S}=\left(\begin{array}{cc}
0 & \mathrm{e}^{\mathrm{j} \varphi_{1}} \\
\mathrm{e}^{\mathrm{j} \varphi_{2}} & 0
\end{array}\right)
$$

Note that the scattering matrix of the waveguide is unitary since we neglected the losses. In order to verify the scattering matrix of the above equation, we perform numerical simulations using the aeroacoustics module of Comsol Multiphysics, which is a full-wave solver working based on finite element method (FEM). In our numerical simulations, we surround the waveguide by sound hard wall boundary conditions, and terminate it at plane wave scattering boundary conditions inducing the excitation. We further assume the material filling the waveguide to be air with a density of $\rho_{0}=1.2 \mathrm{~kg} / \mathrm{m}^{3}$ and the bulk modulus of $K=142 \mathrm{kPa}$. Note that the choice of the filling medium is only for the sake of simplicity. We further remark that in the following, we neglect the Poiseuille flow originated from non-slippery walls, as our simulations show that its effect is indeed negligible. To measure the scattering parameters of the structure, we excite the waveguide from one end with a plane-wave sound field of unit amplitude and measure the phase of the signal receiving at the other end. Figure $2 b$ shows the phase of the corresponding scattering parameters as a function of the fluid 
velocity. The results of this figure show a perfect agreement with those of Figure 2a, which were obtained by a simple argument based on Doppler shifts.
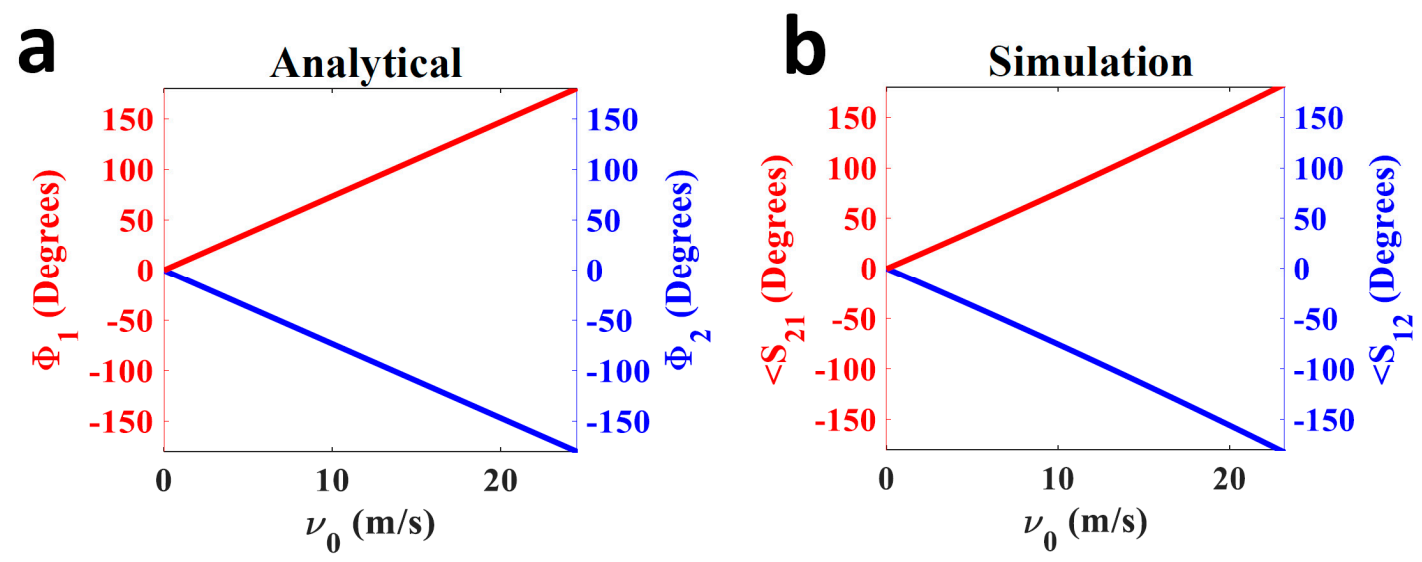

Figure 2. Scattering parameters of the proposed non-reciprocal acoustic phase shifters. (a) Employing Doppler spectral analysis, the variations of $\varphi_{1}$ and $\varphi_{2}$ versus the bias velocity $v_{0}$ are calculated. When the fluid inside the waveguide is stationary, the forward and backward waves are subject to the same amount of phase shift $\left(\varphi_{1}=\varphi_{2}=0\right)$. However, they start acquiring different amounts of transmission phase shifts $\left(\varphi_{1} \neq \varphi_{2}\right)$ by increasing the bias velocity from zero. (b) Variation of the phase of the scattering parameters $\left(S_{12}\right.$ and $\left.S_{21}\right)$ of the structure versus the bias velocity, calculated via full-wave numerical simulations. Numerical simulations validate our Doppler spectral analysis.

To further assess the non-reciprocal behavior of the proposed device, we perform finite difference time domain (FDTD) simulation with a time step of $10^{-5} \mathrm{~s}$. The insets of Figure 3a represent the excitation (solid blue line) and the transmitted (dashed red line) signals when the waveguide is excited from the left side and when a stationary fluid motion is considered $\left(v_{0}=0\right)$. As observed, the injected signal is received after a time delay of $\Delta t=\frac{L}{c}=2.1 \mathrm{~ms}$. However, the received signal is still in phase with the excitation since the waveguide is multiple-wavelengths long. Now assume we impart a bias velocity of $v_{0}=23 \mathrm{~m} / \mathrm{s}$ to the fluid within the waveguide, corresponding to an additional Doppler phase shift of 180 degrees. Figure $3 \mathrm{~b}$ represents the time profiles of the excitation and transmitted fields. As expected, the transmitted signal is led by 180 degrees with respect to the excitation. The promising feature of the device, however, relies not on this phase shift, but on the non-reciprocal phase shift that it provides for the backward propagation. Hence, we repeat our analysis when the waveguide is excited from the other side. Figure $3 c, d$ depict the temporal evolution of the corresponding excitation and transmitted signals in the cases of $v_{0}=0$ and $v_{0}=23 \mathrm{~m} / \mathrm{s}$, respectively. We notice that, when $v_{0}=0$, the transmitted field had exactly the same time profile as that of Figure 3 a. It is because of the fact that, as previously explained, the structure is reciprocal for the stationary fluid velocity. When $v_{0}=23 \mathrm{~m} / \mathrm{s}$, however, the transmitted field is lagged (not led) by 180 degrees with respect to the excitation, clearly confirming the non-reciprocity of the structure. Such simple non-reciprocal phase shifters can be utilized as the building blocks for the synthesis of more complex non-reciprocal devices such as gyrators, isolators, and circulators, among which the realization of the gyrator is discussed in detail in the following section. 
a

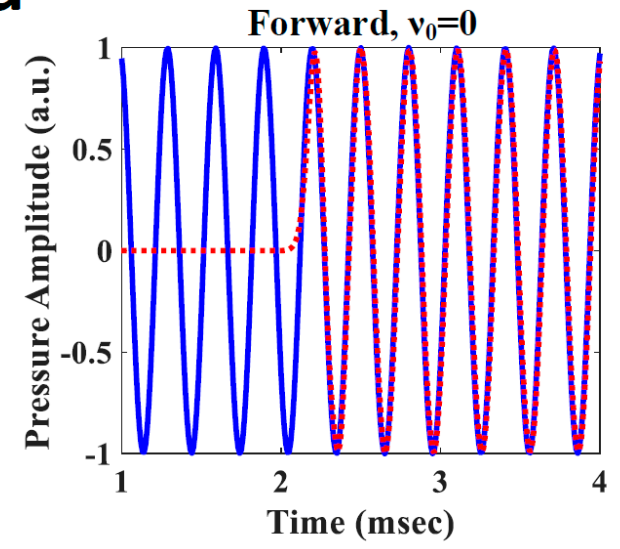

C

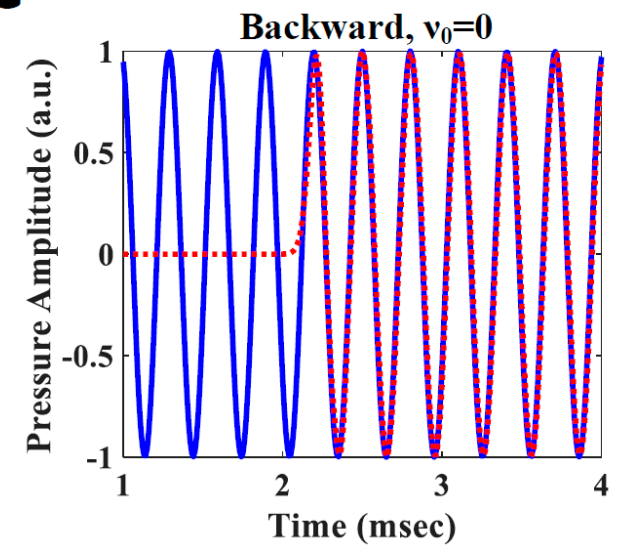

b
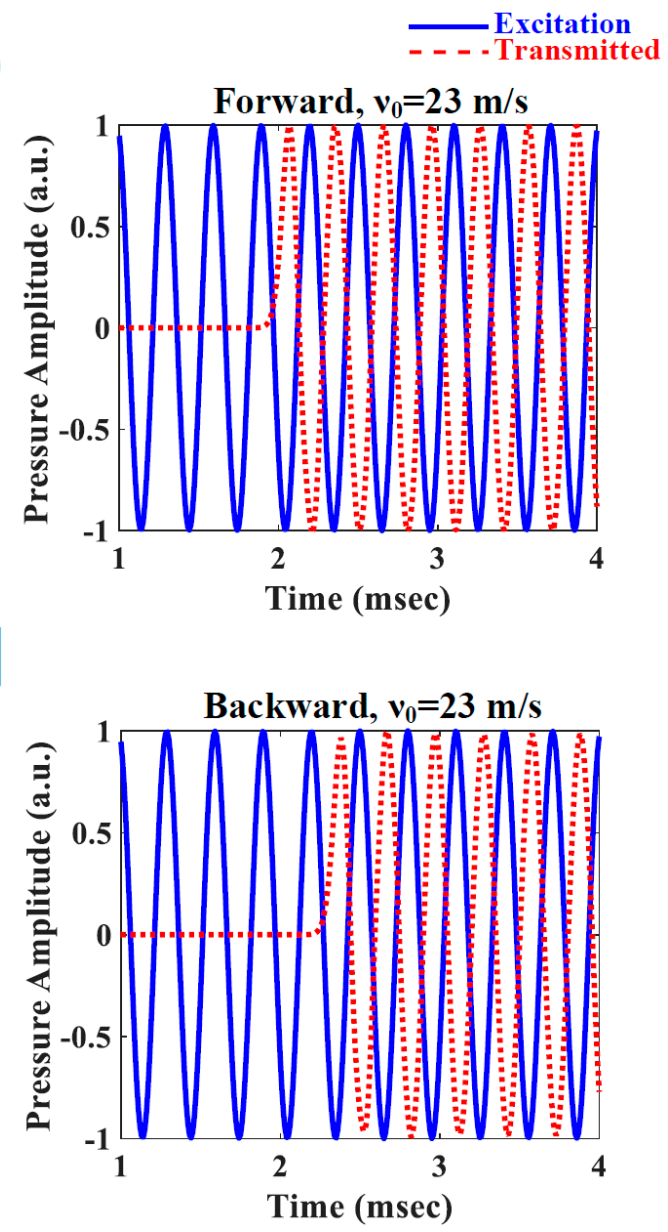

Figure 3. Demonstration of the proposed non-reciprocal acoustic phase shifter. (a) An excitation pulse (the solid blue line) is considered to be injected to the left side of the waveguide of Figure $1 \mathrm{~b}$. The signal that is received at the other side (the red dashed line) is in phase with the excitation when $v_{0}=0$. (b) Same as panel a, except that a bias velocity of $v_{0}=23 \mathrm{~m} / \mathrm{s}$, which corresponds to the phase shift of $\varphi_{1}=\pi$, is imparted on the fluid. The transmitted signal is now led by 180 degrees with respect to the excitation. (c,d) The same process has been followed when the waveguide is excited from the right side. In the case of the stationary fluid (panel c), the time evolution of the transmitted signal is exactly identical to that of panel a, stemming from the reciprocity of the structure. When assuming $v_{0}=23 \mathrm{~m} / \mathrm{s}$, however, the received signal is lagged (not led) by 180 degrees with respect to the input field, manifesting the non-reciprocity of the structure in phase.

\section{Acoustic Gyrator}

This section describes how an acoustic gyrator can be implemented making use of the non-reciprocal phase shifter that was demonstrated in the previous section. A gyrator is a fully passive, lossless two port device. Over the past decades, gyrators have been utilized not only for the network realization of two port components, but also as the building blocks of microwave filters, isolators, impedance converters, and equalizers [1]. The scattering matrix of an ideal gyrator is of the form

$$
\mathrm{S}=\left(\begin{array}{ll}
0 & 1 \\
-1 & 0
\end{array}\right)
$$

which means that a gyrator transmits forward waves without any phase shift, whereas it shifts the phase of backward waves by 180 degrees. Suppose now that the velocity of the fluid filling the waveguide of Figure 1a is chosen such that it corresponds to a phase shift of 90 degrees for forward, 
but that of -90 degrees for backward propagation (Figure 4a). The scattering matrix of the resulting phase shifter then reduces to

$$
\mathrm{S}=\mathrm{e}^{\mathrm{j} \frac{\pi}{2}}\left(\begin{array}{ll}
0 & 1 \\
-1 & 0
\end{array}\right)
$$

which is quite similar to the scattering matrix of an ideal gyrator, apart from a phase factor of $\mathrm{e}^{\mathrm{j} \frac{\pi}{2}}$. This constant phase factor, however, should not cause any concern as it can always be compensated by cascading the phase shifter with a regular reciprocal system that shifts the phase of the travelling waves by -90 degrees for both propagation directions. Hence, our non-reciprocal phase shifter indeed works as an acoustic gyrator for this choice of bias velocity. To prove this, we again perform time domain simulations, assuming a bias velocity motion of $v_{0}=12 \mathrm{~m} / \mathrm{s}$. Figure $4 \mathrm{~b}$ depicts the transmitted signals corresponding to the forward (the solid red line) and backward (the solid blue line) propagation. Notice that the excitation signal is also marked with the dashed black line in the figure. It is obvious that there exists a differential transmission phase shift of 180 degrees between the forward and backward waves. It is further observed that the transmitted signal is led (lagged) by 90 degrees with respect to the excitation for forward (backward) propagation. These obviations are in accordance with the scattering matrix of Equation (3). The pressure field distributions corresponding to the forward and backward transmissions are also depicted in Figure $4 \mathrm{c}, \mathrm{d}$, respectively. The obtained results demonstrate how the proposed phase shifter realizes a gyrator for this choice of bias velocity.
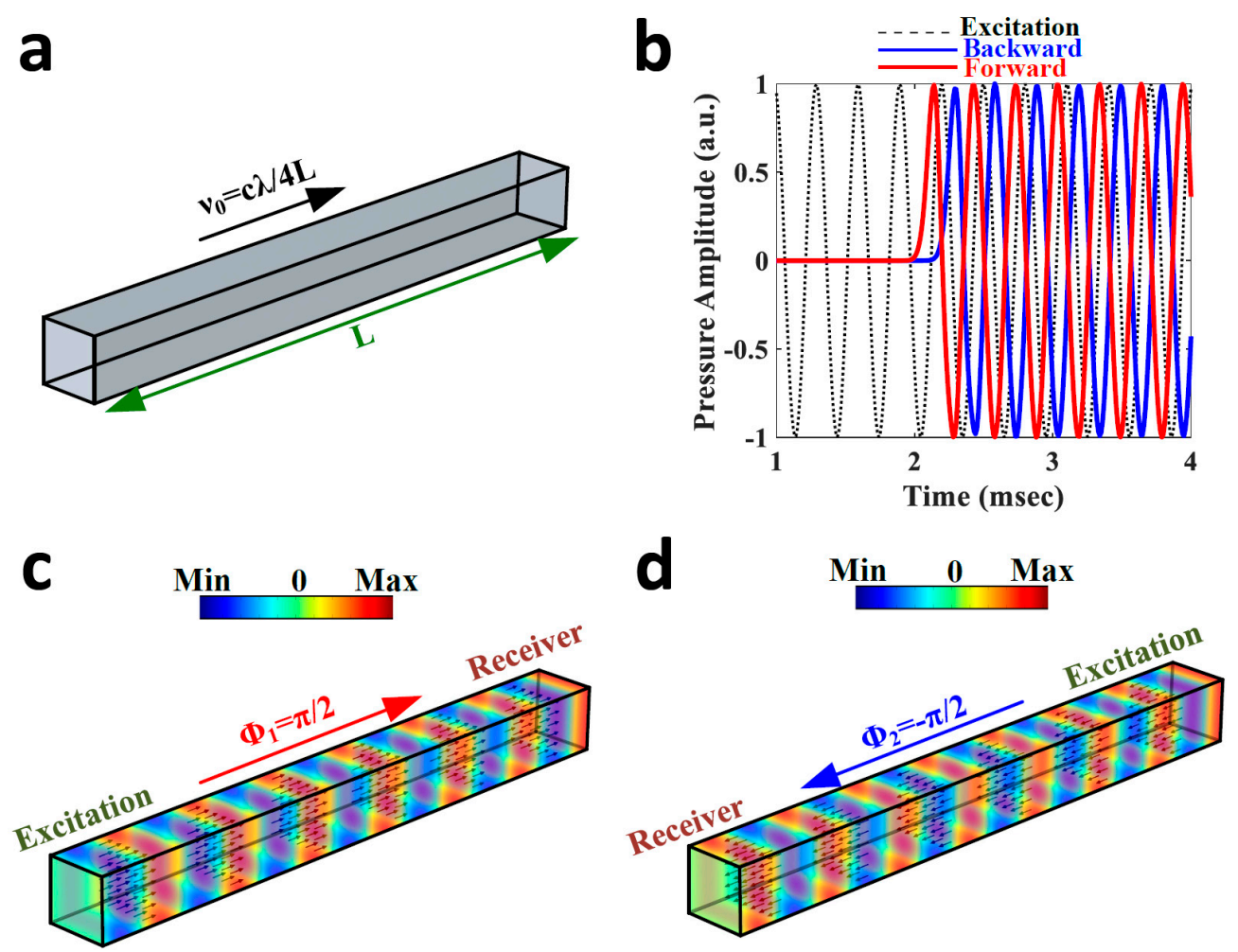

Figure 4. Demonstration of an acoustic gyrator via the proposed non-reciprocal acoustic phase shifter. (a) Bias velocity motion is set such that it corresponds to a transmission phase shift of $\varphi_{1}=\pi / 2$ for forward, and that of $\varphi_{2}=-\pi / 2$ for backward direction. (b) Time profile of the transmitted signals associated with forward (solid red line) and backward (solid blue line) waves. The excitation signal is also marked with a dashed black line. (c,d) Pressure filed distribution corresponding to the forward and backward transmissions. The obtained results reveal that the acoustic phase shifter under study works as a gyrator for this choice of bias velocity. 


\section{Conclusions}

In summary, we proposed a non-reciprocal acoustic phase shifter, providing different phase shifts for the forward and backward propagation of sound waves. To this end, we considered a simple acoustic waveguide that was filled with air. When the air within the waveguide was stationary, the signals travelling inside the waveguide experienced the same amount of phase shift regardless of the propagation direction. However, when a constant bias velocity was applied to the air, the structure became non-reciprocal and the sound waves travelling in opposite directions were subjected to different phase shifts. We further showed that, by changing the amount of bias velocity, one can tune the corresponding differential phase shift. Setting the differential phase shift to 180 degrees, we then realized an acoustic gyrator.

In comparison with the proposals leveraging nonlinear effects to induce non-reciprocity for acoustic waves, the main advantage of our scheme is that it is a fully linear. While non-reciprocal acoustic devices based on non-linearities only work for certain (high) levels of input power, the proposed non-reciprocal acoustic phase shifters and the gyrator work properly regardless of the amount of input power. Such linear non-reciprocal devices may have potential applications to realize various miniaturized and efficient components including isolators, circulators, band pass filters, power controllers, and modulators to mention a few.

Author Contributions: F.Z.-N. carried out the research work under the supervision of R.F. Both authors participated in writing the manuscript.

Funding: This research was funded by Swiss National Science Foundation (SNSF) under Grant No. 172487.

Conflicts of Interest: The authors declare no competing interests.

\section{References}

1. Pozar, D. Microwave Engineering, 4th ed.; John Wiley \& Sons: Hoboken, NJ, USA, 2005; ISBN 9780470631553.

2. Tatarenko, A.S.; Srinivasan, G.; Bichurin, M.I. Magnetoelectric microwave phase shifter. Appl. Phys. Lett. 2006, 88, 183507. [CrossRef]

3. How, H.; Vittoria, C. Microwave phase shifter utilizing nonreciprocal wave propagation. IEEE Trans. Microw. Theory Tech. 2004, 52, 1813-1819. [CrossRef]

4. Babbitt, R.; Koscica, T.; Drach, W.; Didomenico, L. Ferroelectric phase shifters and their performance in microwave phased array antennas. Integr. Ferroelectr. 1995, 8, 65-76. [CrossRef]

5. Hashemi, H.; Guan, X.; Komijani, A.; Hajimiri, A. A 24-GHz SiGe phased-array receiver-LO phase-shifting approach. IEEE Trans. Microw. Theory Tech. 2005, 53, 614-625. [CrossRef]

6. Nikfalazar, M.; Sazegar, M.; Mehmood, A.; Wiens, A.; Friederich, A.; Maune, H.; Binder, J.R.; Jakoby, R. Two-Dimensional Beam-Steering Phased-Array Antenna with Compact Tunable Phase Shifter Based on BST Thick Films. IEEE Antennas Wirel. Propag. Lett. 2017, 16, 585-588. [CrossRef]

7. Akiba, S.; Oishi, M.; Nishikawa, Y.; Minoguchi, K.; Hirokawa, J.; Ando, M. Photonic architecture for beam forming of RF phased array antenna. In Proceedings of the Conference on Optical Fiber Communication, Technical Digest Series, San Francisco, CA, USA, 9-13 March 2014.

8. Creath, K. V Phase-Measurement Interferometry Techniques. Prog. Opt. 1988, 26, 349-393. [CrossRef]

9. Woehrle, C.D.; Doyle, D.T.; Lane, S.A.; Christodoulou, C.G. Space Radiation Environment Testing of Liquid Crystal Phase Shifter Devices. IEEE Antennas Wirel. Propag. Lett. 2016, 15, 1923-1926. [CrossRef]

10. Yan, Y.; Yao, J. A tunable photonic microwave filter with a complex coefficient using an optical RF phase shifter. IEEE Photonics Technol. Lett. 2007, 19, 1472-1474. [CrossRef]

11. Chan, E.H.W.; Minasian, R.A. Photonic RF phase shifter and tunable photonic RF notch filter. J. Lightwave Technol. 2006, 24, 2676-2682. [CrossRef]

12. Liao, L.; Samara-Rubio, D.; Morse, M.; Liu, A.; Hodge, D.; Rubin, D.; Keil, U.D.; Franck, T. High speed silicon Mach-Zehnder modulator. Opt. Express 2005, 13, 3129. [CrossRef] [PubMed]

13. Dong, P.; Chen, L.; Chen, Y. High-speed low-voltage single-drive push-pull silicon Mach-Zehnder modulators. Opt. Express 2012, 20, 6163. [CrossRef] [PubMed] 
14. Roh, Y.S.; Moon, Y.J.; Park, J.; Yoo, C. A two-phase interleaved power factor correction boost converter with a variation-tolerant phase shifting technique. IEEE Trans. Power Electron. 2014, 29, 1032-1040. [CrossRef]

15. Pagani, M.; Marpaung, D.; Eggleton, B.J. Ultra-wideband microwave photonic phase shifter with configurable amplitude response. Opt. Lett. 2014, 39, 5854-5857. [CrossRef] [PubMed]

16. Cheng, Y.-Y.; Wyant, J.C. Phase shifter calibration in phase-shifting interferometry. Appl. Opt. 1985, $24,3049$. [CrossRef] [PubMed]

17. Tarasov, N.; Perego, A.M.; Churkin, D.V.; Staliunas, K.; Turitsyn, S.K. Mode-locking via dissipative Faraday instability. Nat. Commun. 2016, 7, 12441. [CrossRef] [PubMed]

18. Hamoir, G.; De La Torre Medina, J.; Piraux, L.; Huynen, I. Self-biased nonreciprocal microstrip phase shifter on magnetic nanowired substrate suitable for gyrator applications. IEEE Trans. Microw. Theory Tech. 2012, 60, 2152-2157. [CrossRef]

19. Yanaga, M.; Shoji, Y.; Takamura, Y.; Nakagawa, S.; Mizumoto, T. Compact magnetooptical isolator with cobalt ferrite on silicon photonic circuits. Appl. Phys. Express 2015, 8, 082201. [CrossRef]

20. Palomba, M.; Palombini, D.; Colangeli, S.; Ciccognani, W.; Limiti, E. Broadband Nonreciprocal Phase Shifter Design Technique. IEEE Trans. Microw. Theory Tech. 2018, 66, 1964-1972. [CrossRef]

21. Khanikaev, A.B.; Fleury, R.; Mousavi, S.H.; Alù, A. Topologically robust sound propagation in an angular-momentum-biased graphene-like resonator lattice. Nat. Commun. 2015, 6, 8260. [CrossRef] [PubMed]

22. Fleury, R.; Sounas, D.L.; Sieck, C.F.; Haberman, M.R.; Alù, A. Sound isolation and giant linear nonreciprocity in a compact acoustic circulator. Science 2014, 343, 516-519. [CrossRef] [PubMed]

23. He, C.; Ni, X.; Ge, H.; Sun, X.C.; Chen, Y.B.; Lu, M.H.; Liu, X.P.; Chen, Y.F. Acoustic topological insulator and robust one-way sound transport. Nat. Phys. 2016, 12, 1124-1129. [CrossRef]

24. Shen, Z.; Zhang, Y.L.; Chen, Y.; Zou, C.L.; Xiao, Y.F.; Zou, X.B.; Sun, F.W.; Guo, G.C.; Dong, C.H. Experimental realization of optomechanically induced non-reciprocity. Nat. Photonics 2016, 10, 657-661. [CrossRef]

25. Zhang, Z.; Koroleva, I.; Manevitch, L.I.; Bergman, L.A.; Vakakis, A.F. Nonreciprocal acoustics and dynamics in the in-plane oscillations of a geometrically nonlinear lattice. Phys. Rev. E 2016, 94, 032214. [CrossRef] [PubMed]

26. Coulais, C.; Sounas, D.; Alù, A. Static non-reciprocity in mechanical metamaterials. Nature 2017, 542, 461-464. [CrossRef] [PubMed]

27. Zhang, J.; Peng, B.; Özdemir, Ş.K.; Liu, Y.X.; Jing, H.; Lü, X.Y.; Liu, Y.L.; Yang, L.; Nori, F. Giant nonlinearity via breaking parity-time symmetry: A route to low-threshold phonon diodes. Phys. Rev. B 2015, 92, 115407. [CrossRef]

28. Merkel, A.; Willatzen, M.; Christensen, J. Dynamic Nonreciprocity in Loss-Compensated Piezophononic Media. Phys. Rev. Appl. 2018, 9, 034033. [CrossRef]

29. Wang, Q.; Yang, Y.; Ni, X.; Xu, Y.L.; Sun, X.C.; Chen, Z.G.; Feng, L.; Liu, X.P.; Lu, M.H.; Chen, Y.F. Acoustic asymmetric transmission based on time-dependent dynamical scattering. Sci. Rep. 2015, 5, 10880. [CrossRef] [PubMed]

30. Popa, B.I.; Cummer, S.A. Non-reciprocal and highly nonlinear active acoustic metamaterials. Nat. Commun. 2014, 5, 3398. [CrossRef] [PubMed]

31. Li, N.; Ren, J. Non-reciprocal geometric wave diode by engineering Asymmetric shapes of nonlinear materials. Sci. Rep. 2014, 4, 6228. [CrossRef] [PubMed]

32. Fleury, R.; Sounas, D.L.; Haberman, M.R.; Alù, A. Nonreciprocal Acoustics. Acoust. Today 2015, 11, 14.

33. Blanchard, A.; Sapsis, T.P.; Vakakis, A.F. Non-reciprocity in nonlinear elastodynamics. J. Sound Vib. 2018, 412, 326-335. [CrossRef]

34. Fleury, R.; Sounas, D.; Alù, A. An invisible acoustic sensor based on parity-time symmetry. Nat. Commun. 2015, 6, 5905. [CrossRef] [PubMed]

35. Fleury, R.; Khanikaev, A.B.; Alù, A. Floquet topological insulators for sound. Nat. Commun. 2016, 7, 11744. [CrossRef] [PubMed]

36. Zangeneh-Nejad, F.; Fleury, R. Acoustic Analogues of High-Index Optical Waveguide Devices. arXiv, 2018.

37. Zangeneh-Nejad, F.; Fleury, R. Performing Mathematical Operations using High-Index Acoustic Metamaterials. arXiv, 2018.

38. Esfahlani, H.; Karkar, S.; Lissek, H.; Mosig, J.R. Acoustic carpet cloak based on an ultrathin metasurface. Phys. Rev. B 2016, 94, 014302. [CrossRef] 
39. Fleury, R.; Alù, A. Extraordinary sound transmission through density-near-zero ultranarrow channels. Phys. Rev. Lett. 2013, 111, 055501. [CrossRef] [PubMed]

40. Fleury, R.; Alù, A. Metamaterial buffer for broadband non-resonant impedance matching of obliquely incident acoustic waves. J. Acoust. Soc. Am. 2014, 136, 2935-2940. [CrossRef] [PubMed]

41. Landi, M.; Zhao, J.; Prather, W.E.; Wu, Y.; Zhang, L. Acoustic Purcell Effect for Enhanced Emission. Phys. Rev. Lett. 2018, 120, 114301. [CrossRef] [PubMed]

42. Ulbricht, R.; Sakuma, H.; Imade, Y.; Otsuka, P.H.; Tomoda, M.; Matsuda, O.; Kim, H.; Park, G.W.; Wright, O.B. Elucidating gigahertz acoustic modulation of extraordinary optical transmission through a two-dimensional array of nano-holes. Appl. Phys. Lett. 2017, 110, 091910. [CrossRef]

43. Wang, P.; Lu, L.; Bertoldi, K. Topological Phononic Crystals with One-Way Elastic Edge Waves. Phys. Rev. Lett. 2015, 115, 104302. [CrossRef] [PubMed]

44. Li, Y.; Jiang, X.; Li, R.Q.; Liang, B.; Zou, X.Y.; Yin, L.L.; Cheng, J.C. Experimental realization of full control of reflected waves with subwavelength acoustic metasurfaces. Phys. Rev. Appl. 2014, 2, 064002. [CrossRef]

45. Cheng, Y.; Zhou, C.; Yuan, B.G.; Wu, D.J.; Wei, Q.; Liu, X.J. Ultra-sparse metasurface for high reflection of low-frequency sound based on artificial Mie resonances. Nat. Mater. 2015, 14, 1013-1019. [CrossRef] [PubMed]

46. Ni, X.; He, C.; Sun, X.C.; Liu, X.P.; Lu, M.H.; Feng, L.; Chen, Y.F. Topologically protected one-way edge mode in networks of acoustic resonators with circulating air flow. New J. Phys. 2015, 17, 053016. [CrossRef]

47. Wang, W.; Xie, Y.; Konneker, A.; Popa, B.I.; Cummer, S.A. Design and demonstration of broadband thin planar diffractive acoustic lenses. Appl. Phys. Lett. 2014, 105, 101904. [CrossRef]

48. Zhu, Y.F.; Zou, X.Y.; Liang, B.; Cheng, J.C. Acoustic one-way open tunnel by using metasurface. Appl. Phys. Lett. 2015, 107, 113501. [CrossRef]

49. Chen, Y.; Liu, H.; Reilly, M.; Bae, H.; Yu, M. Enhanced acoustic sensing through wave compression and pressure amplification in anisotropic metamaterials. Nat. Commun. 2014, 5, 5247. [CrossRef] [PubMed]

50. Ni, X.; Wu, Y.; Chen, Z.G.; Zheng, L.Y.; Xu, Y.L.; Nayar, P.; Liu, X.P.; Lu, M.H.; Chen, Y.F. Acoustic rainbow trapping by coiling up space. Sci. Rep. 2014, 4, 7038. [CrossRef] [PubMed]

51. Devaux, T.; Tournat, V.; Richoux, O.; Pagneux, V. Asymmetric Acoustic Propagation of Wave Packets Via the Self-Demodulation Effect. Phys. Rev. Lett. 2015, 115, 234301. [CrossRef] [PubMed]

52. Zangeneh-Nejad, F.; Fleury, R. Acoustic Birefringence via Non-Eulerian Metamaterials. arXiv, 2018.

53. Zhai, S.; Chen, H.; Ding, C.; Shen, F.; Luo, C.; Zhao, X. Manipulation of transmitted wave front using ultrathin planar acoustic metasurfaces. Appl. Phys. A Mater. Sci. Process. 2015, 120, 1283-1289. [CrossRef]

54. Popa, B.I.; Shinde, D.; Konneker, A.; Cummer, S.A. Active acoustic metamaterials reconfigurable in real time. Phys. Rev. B 2015, 91, 220303. [CrossRef]

55. Wang, X.P.; Wan, L.L.; Chen, T.N.; Liang, Q.X.; Song, A.L. Broadband acoustic diode by using two structured impedance-matched acoustic metasurfaces. Appl. Phys. Lett. 2016, 109, 044102. [CrossRef]

56. Li, Y.; Shen, C.; Xie, Y.; Li, J.; Wang, W.; Cummer, S.A.; Jing, Y. Tunable asymmetric transmission via lossy acoustic metasurfaces. Phys. Rev. Lett. 2017, 119, 035501. [CrossRef] [PubMed]

57. Huang, Y.L.; Sun, H.X.; Xia, J.P.; Yuan, S.Q.; Ding, X.L. Multi-band asymmetric acoustic transmission in a bended waveguide with multiple mechanisms. Appl. Phys. Lett. 2016, 109, 013501. [CrossRef]

58. Sun, H.; Zhang, S.; Yuan, S.; Xia, J. Experimental verification of manipulating propagation directions of transmitted waves in asymmetric acoustic transmission. Appl. Phys. A Mater. Sci. Process. 2016, 122, 328. [CrossRef]

59. Song, K.; Kim, J.; Hur, S.; Kwak, J.H.; Lee, S.H.; Kim, T. Directional Reflective Surface Formed via Gradient-Impeding Acoustic Meta-Surfaces. Sci. Rep. 2016, 6, 32300. [CrossRef] [PubMed]

60. Ouyang, S.; He, H.; He, Z.; Deng, K.; Zhao, H. Acoustic one-way mode conversion and transmission by sonic crystal waveguides. J. Appl. Phys. 2016, 120, 104504. [CrossRef]

61. Zhang, S.; Zhang, Y.; Guo, Y.; Leng, Y.; Feng, W.; Cao, W. Realization of Subwavelength Asymmetric Acoustic Transmission Based on Low-Frequency Forbidden Transmission. Phys. Rev. Appl. 2016, 5, 034006. [CrossRef]

62. Fan, X.; Qiu, C.; Zhang, S.; Ke, M.; Liu, Z. Highly asymmetric interaction forces induced by acoustic waves in coupled plate structures. J. Appl. Phys. 2015, 118, 244506. [CrossRef]

63. Xia, J.P.; Sun, H.X.; Yuan, S.Q. Modulating Sound with Acoustic Metafiber Bundles/639/301/1005/639/ 766/25/3927/128 article. Sci. Rep. 2017, 7, 8151. [CrossRef] [PubMed] 
64. Zhu, Y.F.; Gu, Z.M.; Liang, B.; Yang, J.; Yang, J.; Yin, L.L.; Cheng, J.C. Asymmetric sound transmission in a passive non-blocking structure with multiple ports. Appl. Phys. Lett. 2016, 109, 103504. [CrossRef]

65. Zhang, Z.; Tian, Y.; Cheng, Y.; Wei, Q.; Liu, X.; Christensen, J. Topological Acoustic Delay Line. Phys. Rev. Appl. 2018, 9. [CrossRef]

66. Shen, C.; Xie, Y.; Li, J.; Cummer, S.A.; Jing, Y. Acoustic metacages for sound shielding with steady air flow. J. Appl. Phys. 2018, 123, 034032. [CrossRef]

67. Zuo, C.Y.; Xia, J.P.; Sun, H.X.; Ge, Y.; Yuan, S.Q.; Liu, X.J. Broadband acoustic logic gates in a circular waveguide with multiple ports. Appl. Phys. Lett. 2017, 111, 243501. [CrossRef]

(c)

(C) 2018 by the authors. Licensee MDPI, Basel, Switzerland. This article is an open access article distributed under the terms and conditions of the Creative Commons Attribution (CC BY) license (http:/ / creativecommons.org/licenses/by/4.0/). 